\title{
DIVISIBILITY PROPERTIES OF THE FIBONACCI ENTRY POINT
}

\author{
PAUL CUBRE AND JEREMY ROUSE
}

(Communicated by Matthew A. Papanikolas)

\begin{abstract}
For a prime $p$, let $Z(p)$ be the smallest positive integer $n$ so that $p$ divides $F_{n}$, the $n$th term in the Fibonacci sequence. Paul Bruckman and Peter Anderson conjectured a formula for $\zeta(m)$, the density of primes $p$ for which $m \mid Z(p)$ on the basis of numerical evidence. We prove Bruckman and Anderson's conjecture by studying the algebraic group $G: x^{2}-5 y^{2}=1$ and relating $Z(p)$ to the order of $\alpha=(3 / 2,1 / 2) \in G\left(\mathbb{F}_{p}\right)$. We are then able to use Galois theory and the Chebotarev density theorem to compute $\zeta(m)$.
\end{abstract}

\section{INTRODUCTION AND STATEMENT OF RESUlts}

Let $F_{n}$ denote the Fibonacci sequence defined as usual by $F_{0}=0, F_{1}=1$ and $F_{n}=F_{n-1}+F_{n-2}$ for $n \geq 2$. If $p$ is a prime number, the smallest positive integer $m$ for which $p \mid F_{m}$ is called the rank of appearance or rank of apparition of $p$. We will denote it by $Z(p)$. For example, we have $Z(11)=10$ since $11 \mid F_{10}=55$ is the smallest Fibonacci number that is a multiple of 11 .

It is well-known that for every prime $p, Z(p) \leq p+1$ (in fact, a proof of this follows from Lemma 8 and Lemma 10 in Section 3). In 1913, Carmichael (see [3], Theorem XXI) proved that if $m \neq 1,2,6,12$, then there is a prime number $p$ so that $Z(p)=m$. It is not presently known if there are infinitely many primes $p$ for which $Z(p)=p+1$.

The main question we study is, given a positive integer $m$, how often does $m$ divide $Z(p)$ ? A natural conjecture would be that $Z(p)$ is "random" $\bmod m$ and so the answer should be $1 / m$. However, Lagarias proved in 1985 (see [9] and [10]) that the density of primes $p$ so that $Z(p)$ is even is $2 / 3$. More precisely,

$$
\lim _{x \rightarrow \infty} \frac{\#\{p \leq x: p \text { is prime and } Z(p) \text { is even }\}}{\pi(x)}=\frac{2}{3},
$$

where $\pi(x)$ is the number of primes $\leq x$. Motivated by this work, Bruckman and Anderson in 2] gathered numerical data and conjectured a formula for

$$
\zeta(m):=\lim _{x \rightarrow \infty} M(m, x) / \pi(x),
$$

where $M(m, x)=\#\{p \leq x: p$ is prime and $m \mid Z(p)\}$. Their conjecture is the following.

Received by the editors December 26, 2012.

2010 Mathematics Subject Classification. Primary 11B39; Secondary 11R32, 14G25.

The first author was partially supported by the Wake Forest University Graduate School.

The second author was supported by NSF grant DMS-0901090. 
Conjecture 1 (Conjecture 3.1 of [2]). If $m=q^{e}$ is a prime power (with $e \geq 1$ ), then

$$
\zeta\left(q^{e}\right)=\frac{q^{2-e}}{q^{2}-1} .
$$

For an arbitrary positive integer $m$, we have

$$
\zeta(m)=\rho(m) \prod_{q^{j} \| m} \zeta\left(q^{j}\right) .
$$

where the product is over all prime powers occurring in the prime factorization of $m$, and

$$
\rho(m)= \begin{cases}1 & \text { if } 10 \nmid m, \\ \frac{5}{4} & \text { if } m \equiv 10 \quad(\bmod 20), \\ \frac{1}{2} & \text { if } 20 \mid m .\end{cases}
$$

The main result of the current paper is a proof of this conjecture.

Theorem 2. Conjecture 1 is true for every positive integer $m$.

One consequence of this result is that the numbers $Z(p)$ show a bias toward being composite. For example, if $q$ is prime, then $\zeta(q)=\frac{q}{q^{2}-1} \approx \frac{1}{q}+\frac{1}{q^{2}}$, and so the numbers $Z(p)$ are divisible by $q$ more often than entries in a random sequence.

To prove Theorem 2, we study the algebraic group

$$
G: x^{2}-5 y^{2}=1 \text {. }
$$

This is a twisted torus isomorphic to $\mathbb{G}_{m}$ over $\mathbb{Q}(\sqrt{5})$. The group law is given by $\left(x_{1}, y_{1}\right) *\left(x_{2}, y_{2}\right)=\left(x_{1} x_{2}+5 y_{1} y_{2}, x_{1} y_{2}+x_{2} y_{1}\right)$. We consider the point $\alpha=$ $(3 / 2,1 / 2) \in G(\mathbb{Q})$, and we show (see Lemma 9) that

$$
n \alpha=\overbrace{\alpha+\alpha+\cdots+\alpha}^{n \text { times }}=\left(L_{2 n} / 2, F_{2 n} / 2\right) .
$$

Here $L_{n}$ is the $n$th Lucas number. These are defined by $L_{0}=2, L_{1}=1$ and $L_{n}=L_{n-1}+L_{n-2}$ for $n \geq 2$. In Lemma 10, we use this identity to relate $Z(p)$ to the order of $\alpha \in G\left(\mathbb{F}_{p}\right)$.

Next, we study the density of primes $p$ for which $m$ divides the order of $\alpha \in$ $G\left(\mathbb{F}_{p}\right)$. In the context of untwisted tori, questions of this nature are quite familiar. They were first considered by Hasse in [5] and [6], and very general results of this type are due to Ballot [1] and Moree [13]. Twisted tori of the type mentioned above are considered by Jones and the second author in [8], and in this paper criteria are given that will guarantee that if $\alpha \in G(\mathbb{Q})$ is fixed and $\ell$ is a prime number, then the density of primes $p$ for which $\ell$ divides the order of $\alpha \in G\left(\mathbb{F}_{p}\right)$ exists and equals $\frac{\ell}{\ell^{2}-1}$.

In the present paper, we extend these results to arbitrary positive integers $m$. To make the extension to prime powers, we consider preimages of $\alpha \in G\left(\mathbb{F}_{p}\right)$ under multiplication by $\ell$. We say that $\alpha$ has an $\ell^{n}$ th preimage if there is a $\beta \in G\left(\mathbb{F}_{p}\right)$ so that $\ell^{n} \beta=\alpha$. In Lemma 11, we relate the order of $\alpha$ with the number of preimages of $\alpha$ under the multiplication by $\ell$. Questions of this type are common in arithmetic dynamics, and these have connections with the Diffie-Hellman key exchange protocol (see [4]). In this context, a generator $\alpha \in \mathbb{F}_{q}^{\times}$is chosen and an 
integer $x$ that encodes a message is also chosen. Computing $y=\alpha^{x}$ given $\alpha$ and $x$ is straightforward, but computing $x$ given $\alpha$ and $y$ is quite difficult. (Note that $\alpha$ is a preimage of $y$.)

To study how often $\alpha$ has an $\ell^{n}$ th preimage, we show that there are $\ell^{n}$ elements $P_{n, r}$ of $G(\mathbb{C})$ so that $\ell^{n} P_{n, r}=\alpha$. We let $K_{\ell^{k}}$ be the field obtained by adjoining all of the $x$ - and $y$-coordinates of the $\ell^{k}$ th preimages to $\mathbb{Q}$. Then, we essentially show that there is an $n$th preimage of $\alpha$ in $G\left(\mathbb{F}_{p}\right)$ if and only if the Artin symbol $\left(\frac{K_{\ell^{k}} / \mathbb{Q}}{\mathfrak{p}}\right)$ fixes an $n$th preimage for all prime ideals $\mathfrak{p}$ above $p$. Finally, we compute $\operatorname{Gal}\left(K_{\ell^{k}} / \mathbb{Q}\right)$ and compute the relevant density. As a consequence, we are able to prove another conjecture of Anderson and Bruckman.

Theorem 3 (Conjecture 2.1 of [2]). Let $p$ be a prime and $\epsilon_{p}=\left(\frac{p}{5}\right)$. Given a prime $q$ and integers $x, i$ and $j$ with $i \geq j \geq 0$, let $M(q, x, i, j)$ denote the number of primes $p \leq x$ such that $q^{i} \|\left(p-\epsilon_{p}\right)$ and $q^{j} \| Z(p)$. Then

$$
\zeta(q ; i, j):=\lim _{x \rightarrow \infty} \frac{M(q, x, i, j)}{\pi(x)}= \begin{cases}\frac{q-2}{q-1} & \text { if } i=j=0, \\ q^{-2 i} & \text { if } i \geq 1 \text { and } j=0, \\ \frac{q-1}{q^{2 i-j+1}} & \text { otherwise. }\end{cases}
$$

To handle the case that $m=\prod_{i=1}^{r} \ell_{i}^{s_{i}}$ is composite, we must show that the fields $K_{\ell_{i}^{s_{i}}}, 1 \leq i \leq r$ are (almost) linearly disjoint. The complication in the formula for $\zeta(m)$ arises from the fact that $\mathbb{Q}(\sqrt{5}) \subseteq K_{2^{a}} \cap K_{5^{b}}$.

An outline of the paper is as follows. We review the relevant algebraic number theory in Section 2, In Section 3 we connect the Fibonacci sequence with the arithmetic of $G\left(\mathbb{F}_{p}\right)$ and $Z(p)$ with the order of $\alpha \in G\left(\mathbb{F}_{p}\right)$, and in turn we connect that with the number of preimages. In Section 4 we define a Galois representation and prove that it is surjective (except in the case that 2 and 5 both divide $m$ ). Finally in Section 5 we compute the relevant densities and prove Theorem 3 and Theorem 2 ,

\section{BACKGROUND}

We begin by reviewing some algebraic number theory. For an introduction to these ideas, see [12]. If $L / K$ is a Galois extension of number fields and $\alpha \in L$, define the norm of $\alpha$ to be

$$
N_{L / K}(\alpha)=\prod_{\sigma \in \operatorname{Gal}(L / K)} \sigma(\alpha) .
$$

If $K / \mathbb{Q}$ is a field extension, let $\mathcal{O}_{K}$ be the ring of algebraic integers in $K$. We say that a prime number $p$ ramifies in $K$ if in the factorization

$$
p \mathcal{O}_{K}=\prod_{i=1}^{n} \mathfrak{p}_{i}^{e_{i}}
$$

we have $e_{i}>1$ for some $i$. For a fixed $K$, only finitely many primes $p$ ramify, and those that ramify are precisely those that divide the discriminant $\Delta_{K}$.

Suppose now that $K / \mathbb{Q}$ is a Galois extension and $\mathfrak{p}$ is a prime ideal in $\mathcal{O}_{K}$. We say that $\mathfrak{p}$ is a prime ideal above $p$ if $\mathfrak{p} \cap \mathbb{Z}=(p)$. This implies that $\mathcal{O}_{K} / \mathfrak{p}$ is a finite extension of $\mathbb{F}_{p}$. If $p$ is unramified in $K / \mathbb{Q}$, then there is a unique element $\sigma \in \operatorname{Gal}(K / \mathbb{Q})$ for which

$$
\sigma(\alpha) \equiv \alpha^{p} \quad(\bmod \mathfrak{p})
$$


for all $\alpha \in \mathcal{O}_{K}$. This element is called the Artin symbol of $\mathfrak{p}$ and is denoted $\left(\frac{K / \mathbb{Q}}{\mathfrak{p}}\right)$. Let

$$
\left(\frac{K / \mathbb{Q}}{p}\right)=\left\{\left(\frac{K / \mathbb{Q}}{\mathfrak{p}}\right): \mathfrak{p} \text { is a prime ideal of } \mathcal{O}_{K} \text { above } p\right\} .
$$

This set is a conjugacy class in $\operatorname{Gal}(K / \mathbb{Q})$.

Let $\zeta_{n}=e^{2 \pi i / n}$. It follows from the definition of the Artin symbol that if $p$ is a prime and $\mathfrak{p}$ is a prime ideal above $p$ in $\mathcal{O}_{\mathbb{Q}\left(\zeta_{n}\right)}$, then $\left(\frac{K / \mathbb{Q}}{\mathfrak{p}}\right)\left(\zeta_{n}\right)=\zeta_{n}^{p}$.

We are now ready to state the Chebotarev density theorem. This result is the key tool we will use to compute the densities mentioned in the introduction. To state it, let $\pi(x)$ be the number of primes $\leq x$.

Theorem 4 ([7, page 143). Suppose that $K / \mathbb{Q}$ is a finite Galois extension. If $\mathcal{C} \subset \operatorname{Gal}(K / \mathbb{Q})$ is a conjugacy class, then

$$
\lim _{x \rightarrow \infty} \frac{\#\left\{p \leq x: p \text { is prime and }\left(\frac{K / \mathbb{Q}}{p}\right)=\mathcal{C}\right\}}{\pi(x)}=\frac{|\mathcal{C}|}{|\operatorname{Gal}(K / \mathbb{Q})|}
$$

We will need a standard result in Galois theory. If $K_{1}$ and $K_{2}$ are two subfields of a field $E$, let $\left\langle K_{1}, K_{2}\right\rangle$ be the smallest subfield that contains both $K_{1}$ and $K_{2}$. The following result describes $\operatorname{Gal}\left(\left\langle K_{1}, K_{2}\right\rangle / F\right)$ in terms of $\operatorname{Gal}\left(K_{1} / F\right), \operatorname{Gal}\left(K_{2} / F\right)$ and $\operatorname{Gal}\left(\left(K_{1} \cap K_{2}\right) / F\right)$.

Theorem 5 ([11], Proposition 3.20). Let $K_{1}$ and $K_{2}$ be Galois extensions of a field $F$. Then $K_{1} \cap K_{2}$ and $\left\langle K_{1}, K_{2}\right\rangle$ are both Galois over $F$ and

$$
\operatorname{Gal}\left(\left\langle K_{1}, K_{2}\right\rangle / F\right) \cong\left\{(\sigma, \tau):\left.\sigma\right|_{K_{1} \cap K_{2}}=\left.\tau\right|_{K_{1} \cap K_{2}}\right\} \subseteq \operatorname{Gal}\left(K_{1} / F\right) \times \operatorname{Gal}\left(K_{2} / F\right) .
$$

In particular,

$$
\left|\left\langle K_{1}, K_{2}\right\rangle: F\right|=\frac{\left|K_{1}: F\right|\left|K_{2}: F\right|}{\left|K_{1} \cap K_{2}: F\right|} .
$$

Finally, given a group $G$, we will denote its order by $|G|$. If $g \in G$ is an element, we will denote the order of $g$ by $|g|$. If $n$ is a non-zero integer and $\ell$ is a prime number, we will denote by $\operatorname{ord}_{\ell}(n)$ the highest power of $\ell$ that divides $n$.

\section{Connection between the Fibonacci sequence and $G\left(\mathbb{F}_{p}\right)$}

In this section we will connect the order of the point $\alpha=(3 / 2,1 / 2) \in G\left(\mathbb{F}_{p}\right)$ with $Z(p)$, the smallest positive integer $n$ so that $p \mid F_{n}$. Given a prime number $\ell$ we will also relate $\operatorname{ord}_{\ell}(|\alpha|)$, ord $\ell\left(\left|G\left(\mathbb{F}_{p}\right)\right|\right)$ and the highest positive integer $m$ so that there is a $\beta \in G\left(\mathbb{F}_{p}\right)$ with $\ell^{m} \beta=\alpha$.

If $K$ is a field, let

$$
G(K)=\left\{(x, y) \in K^{2}: x^{2}-5 y^{2}=1\right\} .
$$

The set $G(K)$ becomes an abelian group with the group operation

$$
\left(x_{1}, y_{1}\right) *\left(x_{2}, y_{2}\right)=\left(x_{1} x_{2}+5 y_{1} y_{2}, x_{1} y_{2}+x_{2} y_{1}\right) .
$$

The identity of $G(K)$ is $(1,0)$, and the inverse of $\left(x_{1}, y_{1}\right)$ is $\left(x_{1},-y_{1}\right)$. The group $G$ is a twisted algebraic torus. It becomes isomorphic to $\mathbb{G}_{m}$ over $K(\sqrt{5})$.

Lemma 6. Let $H(K)=\left\{(x, y) \in K^{2}: x y=1\right\}$. If $\sqrt{5} \in K$ and $\operatorname{char}(K) \neq 2$, then

$$
H(K) \cong G(K)
$$


Proof. Let $\phi: G(K) \rightarrow H(K)$ be given by $\phi(x, y)=(x+\sqrt{5} y, x-\sqrt{5} y)$. It is easily checked that $\phi$ is a homomorphism. If $(x, y) \in H(K)$, then

$$
\phi((x+y) / 2,(x-y) / 2 \sqrt{5})=(x, y)
$$

and

$$
((x+y) / 2,(x-y) / 2 \sqrt{5}) \in G(K) .
$$

This shows that $\phi$ is surjective. Then $\operatorname{ker} \phi=\{(x, y) \in G(K): x+\sqrt{5} y=1$ and $x-$ $\sqrt{5} y=1\}$. If $\operatorname{char}(K) \neq 2$, this implies that $\operatorname{ker} \phi=\{(1,0)\}$ and hence $\phi$ is injective.

A simple consequence of this is the following.

Lemma 7. For any prime $p \neq 2,5, G\left(\mathbb{F}_{p}\right)$ is cyclic.

Proof. We must prove this for two cases: $p \equiv 1,4(\bmod 5)$ and $p \equiv 2,3(\bmod 5)$. In the first case, 5 is a square in $\mathbb{F}_{p}$ and Lemma 6 shows that $G\left(\mathbb{F}_{p}\right) \cong \mathbb{F}_{p}^{\times}$, which is cyclic. In the second case, 5 is a square in $\mathbb{F}_{p^{2}}$ and so $G\left(\mathbb{F}_{p}\right) \subseteq G\left(\mathbb{F}_{p^{2}}\right) \cong \mathbb{F}_{p^{2}}^{\times}$. This shows that $G\left(\mathbb{F}_{p}\right)$ is a subgroup of a cyclic group and is hence cyclic.

The following result gives a formula for the order of $G\left(\mathbb{F}_{p}\right)$.

Lemma 8. Suppose that $p \neq 2,5$ is prime. We have

$$
\left|G\left(\mathbb{F}_{p}\right)\right|=\left\{\begin{array}{lll}
p-1 & \text { if } p \equiv 1 \text { or } 4 \quad(\bmod 5), \\
p+1 & \text { if } p \equiv 2 \text { or } 3 \quad(\bmod 5) .
\end{array}\right.
$$

Proof. In the case that $p \equiv 1,4(\bmod 5)$ the previous lemma gives $\left|G\left(\mathbb{F}_{p}\right)\right|=\left|\mathbb{F}_{p}^{\times}\right|=$ $p-1$. However the following proof handles both cases. A line through the point $(1,0)$ intersects the curve $x^{2}-5 y^{2}=1$ in another rational point if and only if the slope is rational; the same argument applies in $\mathbb{F}_{p}$. Such a line has the form $y \equiv m(x-1)(\bmod p)$. Every point in $G\left(\mathbb{F}_{p}\right)$ other than $(1,0)$ lies on one such line. Computing the intersection of this line with $x^{2}-5 y^{2}=1$ shows that $x=-\frac{1+5 m^{2}}{1-5 m^{2}}$ and $y=-\frac{2 m}{1-5 m^{2}}$. This gives rise to a map $f: S \rightarrow G\left(\mathbb{F}_{p}\right)-\{(1,0)\}$ where $S=$ $\left\{m \in \mathbb{F}_{p}: 5 m^{2} \neq 1\right\}$. The argument above shows that this map is surjective, and a straightforward calculation shows that $f$ is injective. It follows that $\left|G\left(\mathbb{F}_{p}\right)=\right| S \mid$. We have that $|S|=p-1$ when $p \equiv 1,4(\bmod 5)$ and $|S|=p+1$ otherwise. This completes the proof.

The next result shows that the Fibonacci and Lucas sequences occur as the coordinates of multiples of $\alpha=(3 / 2,1 / 2) \in G(\mathbb{Q})$.

Lemma 9. We have $n \alpha=\left(\frac{L_{2 n}}{2}, \frac{F_{2 n}}{2}\right)$.

Proof. We prove this by induction on $n$. The base case for $n=1$ is $\alpha=(3 / 2,1 / 2)=$ $\left(L_{2} / 2, F_{2} / 2\right)$. Our induction hypothesis for $n$ is $n \alpha=\left(L_{2 n} / 2, F_{2 n} / 2\right)$. Then $n \alpha * \alpha=$ $\left(L_{2 n} / 2, F_{2 n} / 2\right) *(3 / 2,1 / 2)=\left(\left(3 L_{2 n}+5 F_{2 n}\right) / 4,\left(L_{2 n}+3 F_{2 n}\right) / 4\right)$. Using the identity $5 F_{n}=L_{n+1}+L_{n-1}$ on the first coordinate we get

$$
\begin{aligned}
3 L_{2 n}+5 F_{2 n} & =3 L_{2 n}+L_{2 n+1}+L_{2 n-1} \\
& =3 L_{2 n}+2 L_{2 n+1}-L_{2 n} \\
& =2\left(L_{2 n}+L_{2 n+1}\right)=2 L_{2 n+2} .
\end{aligned}
$$


Then using the identity $L_{n}=F_{n+1}+F_{n-1}$ on the second coordinate we get

$$
\begin{aligned}
L_{2 n}+3 F_{2 n} & =F_{2 n+1}+F_{2 n-1}+3 F_{2 n} \\
& =F_{2 n+1}+F_{2 n+1}-F_{2 n}+3 F_{2 n} \\
& =2\left(F_{2 n+1}+F_{2 n}\right)=2 F_{2 n+2} .
\end{aligned}
$$

Then we have shown $(n+1)(3 / 2,1 / 2)=\left(L_{2 n+2} / 2, F_{2 n+2} / 2\right)$, completing the induction.

The next result connects the order of $\alpha \in G\left(\mathbb{F}_{p}\right)$ with $Z(p)$.

Lemma 10. Let $p \neq 2,5$ be prime. Then

$$
Z(p)= \begin{cases}2|\alpha| & \text { if }|\alpha| \text { is odd, } \\ \frac{1}{2}|\alpha| & \text { if }|\alpha| \equiv 2 \quad(\bmod 4), \text { and } \\ |\alpha| & \text { if }|\alpha| \equiv 0 \quad(\bmod 4) .\end{cases}
$$

Proof. We need a few identities involving the Lucas and Fibonacci sequences:

$$
\begin{array}{r}
L_{n}^{2}-5 F_{n}^{2}=4(-1)^{n}, \\
L_{n}^{2}=L_{2 n}+2(-1)^{n}, \\
F_{2 n}=F_{n} L_{n} .
\end{array}
$$

First we wish to show $p \mid F_{n}$ if and only if $p \mid F_{2 n}$ and $L_{n}^{2} \equiv 4(-1)^{n}(\bmod p)$. Suppose $p \mid F_{n}$. Clearly $p \mid F_{n} L_{n}=F_{2 n}$ and $p \mid 5 F_{n}=L_{n}^{2}-4(-1)^{n}$. In the other direction, if $p \mid F_{2 n}$, either $p \mid F_{n}$ or $p \mid L_{n}$. From $L_{n}^{2} \equiv 4(-1)^{n}(\bmod p)$, we can conclude that $p \nmid L_{n}$. Therefore $p \mid F_{n}$.

Since $L_{n}^{2} \equiv 4(-1)^{n}(\bmod p)$ implies $L_{2 n}+2(-1)^{n} \equiv 4(-1)^{n}(\bmod p)$, we have $L_{2 n} \equiv 2(-1)^{n}(\bmod p)$. Additionally as $n \alpha=\left(L_{2 n} / 2, F_{2 n} / 2\right)$, it is clear that $p \mid F_{2 n}$ and $L_{2 n} \equiv 2(-1)^{n}(\bmod p)$ if and only if $n \alpha \equiv\left((-1)^{n}, 0\right)(\bmod p)$. Therefore we conclude $p \mid F_{n}$ if and only if $n \alpha \equiv\left((-1)^{n}, 0\right)(\bmod p)$.

It follows that $Z(p)$ is the smallest positive integer $n$ for which $n \alpha \equiv$ $\left((-1)^{n}, 0\right)(\bmod p)$. Since $G\left(\mathbb{F}_{p}\right)$ is cyclic, $(-1,0)$ is the unique element in $G\left(\mathbb{F}_{p}\right)$ of order 2. If $|\alpha|$ is odd, then $(-1,0) \notin\langle\alpha\rangle$. Therefore $n$ is even, $|\alpha| \mid n$, and $Z(p)=2|\alpha|$. If $|\alpha|$ is even, then $(-1,0) \in\langle\alpha\rangle$ and $(|\alpha| / 2) \alpha$ has order 2. When $|\alpha| \equiv 2(\bmod 4),(|\alpha| / 2) \alpha \equiv(-1,0) \equiv\left(-1^{|\alpha| / 2}, 0\right)(\bmod p)$ and $Z(p)=|\alpha| / 2$. When $|\alpha| \equiv 0(\bmod 4)$, then $(|\alpha| / 2) \alpha \equiv(-1,0) \not \equiv\left(-1^{|\alpha| / 2}, 0\right)(\bmod p)$. However $|\alpha| \alpha \equiv(1,0) \equiv\left((-1)^{|\alpha|}, 0\right)(\bmod p)$ and $Z(p)=|\alpha|$.

The next lemma relates the order of $\alpha$ with the largest $m$ for which $\alpha$ has an $\ell^{m}$ th preimage. We will be able to detect the number of preimages using Galois theoretic data, and hence determine the order.

Lemma 11. Suppose that $\ell$ and $p$ are prime numbers and $\alpha \in G\left(\mathbb{F}_{p}\right)$. If $\ell \nmid|\alpha|$, then there are infinitely many preimages of $\alpha$ under multiplication by $\ell$. Suppose that $\ell$ divides $|\alpha|$ and there is an $\ell^{m}$ th preimage of $\alpha$ in $G\left(\mathbb{F}_{p}\right)$, but no $\ell^{m+1}$ th preimage. Then

$$
\operatorname{ord}_{\ell}(|\alpha|)=\operatorname{ord}_{\ell}\left(\left|G\left(\mathbb{F}_{p}\right)\right|\right)-m \text {. }
$$

Proof. Let $\alpha \in G\left(\mathbb{F}_{p}\right), H=\langle\alpha\rangle$ and $\phi: H \rightarrow H$ be given by $\phi(x)=\ell x$. We have that $\phi$ is an automorphism of $H$ if and only if $\operatorname{gcd}(\ell,|H|)=1$, and this implies that $\alpha$ has an $\ell^{m}$ th preimage for all $m$ if and only if $\operatorname{ord}_{\ell}(|\alpha|)=0$. 
On the other hand, suppose that $\ell$ divides $|\alpha|$ and write $\alpha=s \gamma$, where $\gamma$ is a generator of the cyclic group $G\left(\mathbb{F}_{p}\right)$. Write $s=\operatorname{gcd}\left(s,\left|G\left(\mathbb{F}_{p}\right)\right|\right) s^{\prime}$ and note that $s^{\prime} \gamma$ is also a generator of $G\left(\mathbb{F}_{p}\right)$. By replacing $\gamma$ with $s^{\prime} \gamma$, we may assume that $s$ divides $\left|G\left(\mathbb{F}_{p}\right)\right|$.

Let $m=\operatorname{ord}_{\ell}(s)$. Then $\left(s / \ell^{m}\right) \gamma$ is an $\ell^{m}$ th preimage of $\alpha$. It is easy to see, however, that if an $\ell^{m+1}$ st preimage $\beta_{m+1}$ of $\alpha$ existed, then its order would be $\ell^{m+1}|\alpha|$, and this does not divide $\left|G\left(\mathbb{F}_{p}\right)\right|$. Finally, the order of $s \gamma$ is $|\alpha|=\frac{\left|G\left(\mathbb{F}_{p}\right)\right|}{s}$ and this gives

$$
\operatorname{ord}_{\ell}(|\alpha|)=\operatorname{ord}_{\ell}\left(\left|G\left(\mathbb{F}_{p}\right)\right|\right)-m
$$

as desired.

\section{Galois theory}

For a positive integer $m$, let $\gamma_{m}=\sqrt[m]{\frac{3+\sqrt{5}}{2}}$. The isomorphism in Lemma 6 shows that if $m$ is a positive integer, there are precisely $m$ elements $P_{m, r} \in G(\mathbb{C})$ with $m P_{m, r}=\alpha$. These are given by

$$
P_{m, r}=\left(\frac{\zeta_{m}^{r} \gamma_{m}+\zeta_{m}^{-r} \gamma_{m}^{-1}}{2}, \frac{\zeta_{m}^{r} \gamma_{m}-\zeta_{m}^{-r} \gamma_{m}^{-1}}{2 \sqrt{5}}\right), \quad 0 \leq r \leq m-1 .
$$

Note that $m\left(P_{m, r}-P_{m, s}\right)=\alpha-\alpha=0$ and so $P_{m, r}-P_{m, s}$ is a point in $G(\mathbb{C})$ with order dividing $m$.

To study the frequency with which $m$ divides $|\alpha|$ in $G\left(\mathbb{F}_{p}\right)$, we need to know how often the points $P_{m, r}$ "live in $G\left(\mathbb{F}_{p}\right)$." More precisely, this means that if $K_{m}$ is the field obtained by adjoining the $x$ - and $y$-coordinates of all the $P_{m, r}$ to $\mathbb{Q}$, we need to determine how often the rational prime $p$ is contained in a prime ideal $\mathfrak{p} \subset \mathcal{O}_{K_{m}}$ so that

$$
\left(\frac{K_{m} / \mathbb{Q}}{\mathfrak{p}}\right)\left(P_{m, r}\right)=P_{m, r}
$$

If the above equation is true, this implies that the point $P_{m, r}$ (which for all but finitely many primes $p$ can be thought of as an element of the finite field $\mathcal{O}_{K_{m}} / \mathfrak{p}$ ) is fixed by a generator of the Galois group of $\mathcal{O}_{K_{m}} / \mathfrak{p}$ over $\mathbb{F}_{p}$; i.e., there is an element $\beta \in G\left(\mathbb{F}_{p}\right)$ so that $m \beta=\alpha$. We must first understand $\operatorname{Gal}\left(K_{m} / \mathbb{Q}\right)$ and its action on the points $\left\{P_{m, r}\right\}$. For simplicity, let

$$
\begin{aligned}
P_{m} & :=P_{m, 0}=\left(\frac{\gamma_{m}+\gamma_{m}^{-1}}{2}, \frac{\gamma_{m}-\gamma_{m}^{-1}}{2 \sqrt{5}}\right), \\
Q_{m} & :=P_{m, 1}-P_{m, 0}=\left(\frac{\zeta_{m}+\zeta_{m}^{-1}}{2}, \frac{\zeta_{m}-\zeta_{m}^{-1}}{2 \sqrt{5}}\right) .
\end{aligned}
$$

Let $I(m)=\left\{a x+b: a \in(\mathbb{Z} / m \mathbb{Z})^{\times}, b \in(\mathbb{Z} / m \mathbb{Z})\right\}$ denote the affine group over $\mathbb{Z} / m \mathbb{Z}$. The group law on $I(m)$ is composition.

Lemma 12. The extension $K_{m} / \mathbb{Q}$ is Galois. For each $\sigma \in \operatorname{Gal}\left(K_{m} / \mathbb{Q}\right)$, there are elements $a_{\sigma} \in(\mathbb{Z} / m \mathbb{Z})^{\times}$and $b_{\sigma} \in(\mathbb{Z} / m \mathbb{Z})$ so that $\sigma\left(P_{m}+r Q_{m}\right)=P_{m}+$ $\left(a_{\sigma} r+b_{\sigma}\right) Q_{m}$ for $0 \leq r \leq m-1$. The map

$$
\rho: \operatorname{Gal}\left(K_{m} / \mathbb{Q}\right) \rightarrow I(m)
$$

given by $\rho(\sigma)=a_{\sigma} x+b_{\sigma}$ is an injective homomorphism. 
Proof. The field $K_{m}$ lies in the Galois extension $\mathbb{Q}\left(\gamma_{m}, \zeta_{m}, \sqrt{5}\right)$ over $\mathbb{Q}$. Then $K_{m}$ is separable as it is an intermediate field of a separable extension, and it is a splitting field as we are adjoining all conjugates of the coordinates $P_{m}$ and $Q_{m}$. We conclude that the extension $K_{m} / \mathbb{Q}$ is Galois. For $\sigma \in \operatorname{Gal}\left(K_{m} / \mathbb{Q}\right)$, $\sigma\left(m P_{m, r}\right)=m \sigma\left(P_{m, r}\right)=\alpha$. Therefore $\operatorname{Gal}\left(K_{m} / \mathbb{Q}\right)$ must take a preimage of $\alpha$ to another preimage of $\alpha$. Hence $\sigma\left(P_{m, 0}\right)=P_{m, b_{\sigma}}=P_{m, 0}+b_{\sigma} Q_{m}$. Additionally $\sigma\left(m Q_{m}\right)=m \sigma\left(Q_{m}\right)=0$. So $\operatorname{Gal}\left(K_{m} / \mathbb{Q}\right)$ must take an element of order $m$ to another element of order $m$. Hence $\sigma\left(Q_{m}\right)=a_{\sigma} Q_{m}$. This yields

$$
\sigma\left(P_{m}+r Q_{m}\right)=\sigma\left(P_{m}\right)+r \sigma\left(Q_{m}\right)=P_{m}+\left(a_{\sigma} r+b_{\sigma}\right) Q_{m} .
$$

From the definition of $\rho$, it is easy to see that $\rho$ is a homomorphism. The kernel of $\rho$ is the set $\left\{\sigma \in \operatorname{Gal}\left(K_{m} / \mathbb{Q}\right): \rho(\sigma)=x\right\}$. If $\sigma \in \operatorname{ker} \rho$, then $\sigma\left(P_{m}+r Q_{m}\right)=P_{m}+r Q_{m}$ for all $r$. This implies that $P_{m}$ and $Q_{m}$ are both fixed by $\sigma$. Since the coordinates of $P_{m}$ and $Q_{m}$ generate $K_{m}$ over $\mathbb{Q}$, it follows that $\sigma$ fixes $K_{m}$ and so ker $\rho=1$.

The Chinese remainder theorem allows one to see that if the prime factorization of $m=\prod_{i=1}^{r} \ell_{i}^{s_{i}}$, then

$$
I(m) \cong \prod_{i=1}^{r} I\left(\ell_{i}^{s_{i}}\right) .
$$

We will therefore begin by studying the images of $\operatorname{Gal}\left(K_{m} / \mathbb{Q}\right) \rightarrow I(m)$ in the case where $m$ is a prime power. This case was studied by Jones and the second author in [8. In particular, Proposition 4.1 and Theorem 4.2 (with the special case $d=5$ ) handle the case where $m=\ell^{k}$ is a prime. Specifically, if $F$ is a number field, the map

$$
\rho: \operatorname{Gal}\left(\left\langle K_{m}, F\right\rangle / F\right) \rightarrow I\left(\ell^{k}\right)
$$

is surjective for all $k$ if and only if

(1) there is no point $\beta \in G(F)$ with $\ell \beta=\alpha$, and

(2) $\left|F\left(\zeta_{\ell^{3}}+\zeta_{\ell^{3}}^{-1}\right): F\right|=\frac{\ell^{2}(\ell-1)}{2}$, and

(3) if $\ell=2,-2$ and -10 are not squares in $F$ and if $L$ is the field obtained by adjoining to $F$ the coordinates of $Q_{8}$, then there is no point $\beta \in G(L)$ with $2 \beta=\alpha$.

Using this result, we deduce the following result concerning the surjective of the $\operatorname{map} \rho: \operatorname{Gal}\left(K_{m} / \mathbb{Q}\right) \rightarrow I(m)$.

Lemma 13. Let $\ell$ be a prime and $k \geq 1$. Then the map $\rho: \operatorname{Gal}\left(K_{\ell^{k}} / \mathbb{Q}\right) \rightarrow I\left(\ell^{k}\right)$ is surjective.

Proof. If there is a $\beta \in G(\mathbb{Q}(\sqrt{5}))$ with $\ell \beta=\alpha$, then the isomorphism $G(\mathbb{Q}(\sqrt{5})) \cong$ $H(\mathbb{Q}(\sqrt{5}))$ from Lemma 6 sends $\beta$ to $\sqrt[\ell]{(3+\sqrt{5}) / 2}$. It is well-known that $\mathcal{O}_{\mathbb{Q}(\sqrt{5})}=$ $\mathbb{Z}\left[\frac{1+\sqrt{5}}{2}\right]$ and $\mathbb{Z}\left[\frac{1+\sqrt{5}}{2}\right]^{\times}$is generated by -1 and $\frac{1+\sqrt{5}}{2}$. Since $\frac{3+\sqrt{5}}{2}=\left(\frac{1+\sqrt{5}}{2}\right)^{2}$, it follows that $\ell=2$. However, the second preimages of $\alpha$ are $\left(\frac{ \pm \sqrt{5}}{2}, \frac{ \pm \sqrt{5}}{10}\right)$, which are not in $G(\mathbb{Q})$. This establishes condition $(1)$. It is well-known that $\mid \mathbb{Q}\left(\zeta_{\ell^{3}}+\right.$ $\left.\zeta_{\ell^{3}}^{-1}\right): \mathbb{Q} \mid=\frac{\ell^{2}(\ell-1)}{2}$, and so condition (2) holds. For condition (3), a simple check shows that $L=\mathbb{Q}(\sqrt{2}, \sqrt{-5})$ and so $\sqrt{5} \notin L$ and thus there is no $\beta \in G(L)$ with $2 \beta=\alpha$. 
The next step is extending the result of the above lemma to prove that the map $\operatorname{Gal}\left(K_{m} / \mathbb{Q}\right) \rightarrow I(m)$ is surjective (unless $10 \mid m$ ).

Lemma 14. If $\ell>2$ is prime, then every minimal subfield of $K_{\ell^{k}} / \mathbb{Q}$ is either $\mathbb{Q}\left(P_{\ell}\right)$ (or some conjugate) or is contained in $\mathbb{Q}\left(\zeta_{\ell^{2}}\right)$. If $\ell=2$, then every minimal subfield of $K_{2^{k}} / \mathbb{Q}$ is contained in $\mathbb{Q}(\sqrt{5}, i, \sqrt{2})$.

Proof. Lemmas 12 and 13 translate this into a group theory problem. Let $N=$ $\left\{a x+b \in I\left(\ell^{k}\right): a \equiv 1(\bmod \ell)\right\}$. This is a normal subgroup of $I\left(\ell^{k}\right)$. If $G$ is a group, let $\Phi(G)$ denote the Frattini subgroup of $G$, the intersection of the maximal subgroups of $G$. Since $N \unlhd G, \Phi(N) \subseteq \Phi(G)$. Since the order of $N$ is a power of $\ell$, every maximal subgroup has index $\ell$; and so if $n \in N$, then $n^{\ell} \in \Phi(N)$. This implies that

$$
\left\{a x+b \in I\left(\ell^{k}\right): a \equiv 1 \quad\left(\bmod \ell^{2}\right), b \equiv 0 \quad(\bmod \ell)\right\} \subseteq \Phi(N) \subseteq \Phi(G),
$$

and, in particular, that the kernel of the map from $I\left(\ell^{k}\right) \rightarrow I\left(\ell^{2}\right)$ is contained in $\Phi(G)$. Since $I\left(\ell^{2}\right)$ is solvable, every maximal subgroup has prime power index. Using the surjective homomorphism $\phi: I\left(\ell^{2}\right) \rightarrow\left(\mathbb{Z} / \ell^{2} \mathbb{Z}\right)^{\times}$, we can identify the maximal subgroups of index less than $\ell$ and also show there are none of index $>\ell$. If $M \subseteq I\left(\ell^{2}\right)$ is a maximal subgroup of index $\ell$, then either $x+1 \in M$, in which case $M=\left\{a x+b: a^{\ell-1} \equiv 1\left(\bmod \ell^{2}\right)\right\}$, or $x+1 \notin M$. Note that if $f_{1}=a x+b$ and $f_{2}=c x+d$, then $f_{1} \circ f_{2} \circ f_{1}^{-1} \circ f_{2}^{-1}=x+a d-b c+b-d$. Hence, if $x+1 \notin M$, then $M$ is abelian. This implies that if $a x+b$ and $c x+d$ are in $M$, then $(a-1) d=(c-1) b$. If $e$ is the common value of $\frac{b}{a-1}$ for $a \neq 1$, then we have

$$
M=\left\{a(x-e)+e: a \in\left(\mathbb{Z} / \ell^{2} \mathbb{Z}\right)^{\times}\right\}
$$

for some $e$ with $0 \leq e \leq \ell^{2}-1$. All of these subgroups are conjugates of $\{a x: a \in$ $\left.\left(\mathbb{Z} / \ell^{2} \mathbb{Z}\right)^{\times}\right\}$. Translating back to fields, we obtain the desired result when $\ell>2$.

When $\ell=2$ we have $\Phi\left(I\left(2^{k}\right)\right)=\{a x+b: a \equiv 1(\bmod 8), b \equiv 0(\bmod 2)\}$ provided $k \geq 3$, and a straightforward calculation shows that the field corresponding to $\Phi\left(I\left(2^{k}\right)\right)$ is $\mathbb{Q}(\sqrt{5}, i, \sqrt{2})$.

Suppose that $\ell_{1}, \ell_{2}, \ldots, \ell_{n}$ are prime divisors of $m$. To prove that $\operatorname{Gal}\left(K_{m} / \mathbb{Q}\right) \rightarrow$ $I(m)$ is surjective, we will show that $K_{\ell_{n}^{s_{n}}} \cap\left\langle K_{\ell_{1}^{s_{1}}}, K_{\ell_{2}^{s_{2}}}, \ldots, K_{\ell_{n-1}}^{s_{n-1}}\right\rangle=\mathbb{Q}$. We will prove this using ramification properties of these fields.

Lemma 15. Suppose that $\ell$ is prime. Then $K_{\ell^{k}} / \mathbb{Q}$ is ramified only at 5 and $\ell$. If $\ell \neq 2$, then every minimal subfield of $K_{\ell^{k}} / \mathbb{Q}$ is ramified at $\ell$.

Proof. From the formulas for the $P_{\ell^{k}, r}$ it is clear that $K_{\ell^{k}}$ is contained in the splitting field of $x^{\ell^{k}}-\frac{3+\sqrt{5}}{2}$ over $\mathbb{Q}(\sqrt{5})$. The discriminant $\Delta_{L / \mathbb{Q}}$ of $L=\mathbb{Q}\left(\gamma_{\ell^{k}}\right)$ is

$$
\Delta_{L / \mathbb{Q}}=\Delta_{\mathbb{Q}(\sqrt{5}) / \mathbb{Q}}^{[L: \mathbb{Q}(\sqrt{5})]} \cdot N_{L / \mathbb{Q}(\sqrt{5})}\left(\Delta_{L / \mathbb{Q}(\sqrt{5})}\right) .
$$

Since the discriminant of $x^{\ell^{k}}-\frac{3+\sqrt{5}}{2}$ is a power of $\ell$ times a unit, it follows that $\Delta_{L / \mathbb{Q}}$ is a power of 5 times a power of $\ell$. If $L_{1}$ and $L_{2}$ are two extensions of $\mathbb{Q}$ ramified only at primes in a set $S$, then $\left\langle L_{1}, L_{2}\right\rangle / \mathbb{Q}$ is ramified only at primes in $S$ (see Theorem 4.67 of [12]). It follows from this that the splitting field of $x^{\ell^{k}}-\frac{3+\sqrt{5}}{2}$ is ramified only at 5 and $\ell$ and hence $K_{\ell^{k}} / \mathbb{Q}$ is too. 
To prove the second claim it is enough, by Lemma 14 to prove that $\mathbb{Q}\left(P_{\ell}\right)$ is ramified at $\ell$. From the surjectivity of $\rho$ proven in Lemma 13, it follows that $\operatorname{Gal}\left(K_{\ell} / \mathbb{Q}\right)$ acts transitively on the $P_{\ell, r}$. Therefore, the Galois closure of $\mathbb{Q}\left(P_{\ell}\right)$ over $\mathbb{Q}$ is $K_{\ell}$. Since $\mathbb{Q}\left(\zeta_{\ell}+\zeta_{\ell}^{-1}\right)$ is ramified at $\ell$ and is contained in $K_{\ell}$, it follows that $K_{\ell} / \mathbb{Q}$ is ramified at $\ell$, and this implies that $\mathbb{Q}\left(P_{\ell}\right)$ is ramified at $\ell$ as well.

Lemma 16. Let $m$ be an arbitrary positive integer. The map $\rho: \operatorname{Gal}\left(K_{m} / \mathbb{Q}\right) \rightarrow$ $I(m)$ is surjective if $10 \nmid m$. If $10 \mid m$, then the image of $\rho$ is

$$
\{a x+b: b \text { is even } \Longleftrightarrow a \equiv 1 \text { or } 4(\bmod 5)\} .
$$

Proof. First note that if $\operatorname{gcd}\left(m_{1}, m_{2}\right)=1$, then $\left\langle K_{m_{1}}, K_{m_{2}}\right\rangle=K_{m_{1} m_{2}}$. It is clear that $K_{m_{1}}, K_{m_{2}} \subseteq K_{m_{1} m_{2}}$. For the reverse direction note that if $m_{1} x+m_{2} y=1$, then

$$
m_{1} m_{2}\left(y P_{m_{1}}+x P_{m_{2}}\right)=m_{2} y \alpha+m_{1} x \alpha=\alpha .
$$

Thus $y P_{m_{1}}+x P_{m_{2}}$ is a preimage of $\alpha$ under multiplication by $m_{1} m_{2}$. Further, since $G\left(\left\langle K_{m_{1}}, K_{m_{2}}\right\rangle\right)$ contains elements of order $m_{1}$ and $m_{2}$, it must contain $Q_{m_{1} m_{2}}$ and so $K_{m_{1} m_{2}}=\left\langle K_{m_{1}}, K_{m_{2}}\right\rangle$.

Suppose first that $10 \nmid m$. To prove that $\rho$ is surjective, we will prove (by induction on the $n$, the number of distinct prime factors of $m)$ that $\left|\operatorname{Gal}\left(K_{m} / \mathbb{Q}\right)\right|=$ $|I(m)|$. The base case $(n=1)$ is handled by Lemma 13 .

Suppose that $m=\prod_{i=1}^{n} \ell_{i}^{s_{i}}$ is the prime factorization of $m$. Since $K_{m / \ell_{n}^{s_{n}}}=$ $\left\langle K_{\ell_{1}^{s_{1}}}, K_{\ell_{2}^{s_{2}}}, \ldots, K_{\ell_{n-1}^{s_{n-1}}}\right\rangle$ we have that $K_{m / \ell_{n}^{s_{n}}}$ is ramified only at 5 and $\ell_{1}, \ldots, \ell_{n-1}$, while $K_{\ell_{n}^{s_{n}}}$ is ramified only at 5 and $\ell_{n}$. Moreover, by Lemma 15 every minimal subfield of $K_{\ell_{n}^{s n}}$ is ramified at $\ell_{n}$. It follows therefore that $K_{\ell_{n}^{s_{n}}} \cap K_{m / \ell_{n}^{s} n}$ is unramified everywhere, and since $\mathbb{Q}$ has no unramified extensions we have that $K_{\ell_{n}^{s_{n}}} \cap K_{m / \ell_{n}^{s_{n}}}=\mathbb{Q}$. From Theorem $[5$ we obtain that

$$
\left|\left\langle K_{\ell_{n}^{s_{n}}}, K_{m / \ell_{n}^{s_{n}}}\right\rangle: \mathbb{Q}\right|=\left|K_{\ell_{n}^{s_{n}}}: \mathbb{Q}\right| \cdot\left|K_{m / \ell_{n}^{s_{n}}}: \mathbb{Q}\right|=\left|I\left(\ell_{n}^{s_{n}}\right)\right| \cdot\left|I\left(m / \ell_{n}^{s_{n}}\right)\right|=|I(m)|,
$$

which proves the desired result by induction on $n$.

A similar argument shows that if $10 \mid m$, then

$$
\left|K_{m}: \mathbb{Q}\right|=\left|K_{2^{s_{1} 5^{s_{2}}}:}: \mathbb{Q}\right| \cdot\left|K_{m /\left(2^{\left.s_{1} 5^{s_{2}}\right)}:\right.}: \mathbb{Q}\right| \text {. }
$$

To determine $\left|K_{2^{s_{1}} 5^{s_{2}}}: \mathbb{Q}\right|$ we will show that $K_{2^{s_{1}}} \cap K_{5^{s_{2}}}=\mathbb{Q}(\sqrt{5})$ by determining the subfields of $K_{2^{s_{1}}}$ that are ramified only at 5 . We have that $\mathbb{Q}(\sqrt{5}) \subseteq K_{2^{s_{1}}}$ and the subgroup of $I\left(2^{s_{1}}\right)$ corresponding to $\mathbb{Q}(\sqrt{5})$ is

$$
H=\{a x+b: b \equiv 0 \quad(\bmod 2)\} .
$$

Since $H$ is a 2-group, $\Phi(H)=H^{\prime} H^{2}=\{a x+b: a \equiv 1(\bmod 8), b \equiv 0(\bmod 4)\}$. The field corresponding to this subgroup is $L=\mathbb{Q}\left(\sqrt{\frac{1+\sqrt{5}}{2}}, i, \sqrt{2}\right)$, and it is straightforward to see that the maximal subextension of $L$ ramified only at 5 is $\mathbb{Q}(\sqrt{5})$. It follows that $K_{2^{s_{1}}} \cap K_{5^{s_{2}}}=\mathbb{Q}(\sqrt{5})$ and so when $10|m,| K_{m}: \mathbb{Q}\left|=\frac{1}{2}\right| I(m) \mid$. Finally, since $P_{2}=\left(\frac{\sqrt{5}}{2}, \frac{\sqrt{5}}{10}\right)$ we have that $\sigma \in \operatorname{Gal}\left(K_{m} / \mathbb{Q}\right)$ fixes $\sqrt{5}$ if and only if it fixes $P_{2}$ and this occurs if and only if $\rho(\sigma)=a x+b$ where $b \equiv 0(\bmod 2)$ and $\left(\frac{a}{5}\right)=1$. This yields the desired result.

Remark. The method from the previous sections is very general and can be used to establish the surjectivity of Galois representations attached to arbitrary onedimensional tori. 


\section{Density computations}

In this section, we will translate conditions on when preimages of $\alpha$ exist in $G\left(\mathbb{F}_{p}\right)$ into statements about the Frobenius conjugacy class $\left(\frac{K_{m} / \mathbb{Q}}{p}\right)$. We will then count the sizes of these classes and use this to prove Theorem 2 . We will start by focusing on the prime power case.

As in the previous section, let $\ell$ be a prime number, $k \geq 1$ and $K_{\ell^{k}}$ be the field obtained by adjoining all the $x$ - and $y$-coordinates of $P_{\ell^{k}, r}$ to $\mathbb{Q}$. Let $\rho$ : $\operatorname{Gal}\left(K_{\ell^{k}} / \mathbb{Q}\right) \rightarrow I\left(\ell^{k}\right)$ be the homomorphism defined in Lemma 12, We define

$$
\mathcal{C}_{k, n, \ell}=\left\{\sigma \in \operatorname{Gal}\left(K_{\ell^{k}} / \mathbb{Q}\right): \sigma\left(\ell^{k-n} P_{\ell^{k}, r}\right)=\ell^{k-n} P_{\ell^{k}, r}\right.
$$

for some $r$ with $\left.0 \leq r \leq \ell^{k}-1\right\}$.

We will make the convention that if $n<0$, then $\mathcal{C}_{k, n, \ell}$ is empty. Since $P_{\ell^{k}, r}$ is an $\ell^{k}$ th preimage of $\alpha, \ell^{k-n} P_{\ell^{k}, r}$ is an $\ell^{n}$ th preimage of $\alpha$.

Lemma 17. Let $p \neq 2,5, \ell$ be a prime number. Then, there is an nth preimage of $\alpha$ in $G\left(\mathbb{F}_{p}\right)$ if and only if $\left(\frac{K_{\ell k} / \mathbb{Q}}{p}\right) \subseteq \mathcal{C}_{k, n, \ell}$.

Proof. First suppose that $\left(\frac{K_{\ell k} / \mathbb{Q}}{p}\right) \subseteq \mathcal{C}_{k, n, \ell}$ and let $\mathfrak{p}$ be a prime ideal above $p$ in $\mathcal{O}_{K_{\ell^{k}}}$. Let $\sigma=\left(\frac{K_{\ell^{k}} / \mathbb{Q}}{\mathfrak{p}}\right)$ fix $\ell^{k-n} P_{\ell^{k}, r}$. We may consider $\ell^{k-n} P_{\ell^{k}, r}$ as an element of $G\left(\mathcal{O}_{K_{\ell k}} / \mathfrak{p}\right)$ (note that 2 and 5 are the only primes that divide the denominators of coordinates of preimages of $\alpha$ ). Since $\sigma$ fixes $\ell^{k-n} P_{\ell^{k}, r}$ and $\sigma$ acts as the Frobenius automorphism on $\mathcal{O}_{K_{\ell k}} / \mathfrak{p}$, it follows that $\ell^{k-n} P_{\ell^{k}, r} \in G\left(\mathbb{F}_{p}\right)$, as desired.

To show the reverse implication we will first show that for any prime $\mathfrak{p}$ above $p$, the reduction mod $\mathfrak{p}$ map on $\ell^{n}$ th preimages of $\alpha$ is injective. Any $n$th preimage of $\alpha$ has the form $P_{\ell^{n}, r}=P_{\ell^{n}}+r Q_{\ell^{n}}$. If $P_{\ell^{n}}+r_{1} Q_{\ell^{n}} \equiv P_{\ell^{n}}+r_{2} Q_{\ell^{n}}(\bmod \mathfrak{p})$ with $r_{1} \not \equiv$ $r_{2}\left(\bmod \ell^{n}\right)$, then $\left(r_{1}-r_{2}\right) Q_{\ell^{n}}$ is congruent to the identity $\bmod \mathfrak{p}$. Every element of order $\ell$ is a multiple of $\left(r_{1}-r_{2}\right) Q_{\ell^{n}}$, and this implies that the $y$-coordinate of $Q_{\ell}$ is $\equiv 0(\bmod \mathfrak{p})$, which implies that

$$
N_{\mathbb{Q}\left(\zeta_{\ell}, \sqrt{5}\right) / \mathbb{Q}}\left(\frac{\zeta_{\ell}-\zeta_{\ell}^{-1}}{2 \sqrt{5}}\right) \equiv 0 \quad(\bmod p) .
$$

This is a contradiction because $N_{\mathbb{Q}\left(\zeta_{\ell}\right) / \mathbb{Q}}\left(\zeta_{\ell}-\zeta_{\ell}^{-1}\right)=\ell$. Hence the reduction $\bmod \mathfrak{p}$ map is injective on $\ell^{n}$ th preimages.

Finally, suppose there is an $\ell^{n}$ th preimage of $\alpha$ in $G\left(\mathbb{F}_{p}\right)$. For a prime ideal $\mathfrak{p}$ above $p$, we consider $\mathbb{F}_{p} \subseteq \mathcal{O}_{K_{\ell^{k}}} / \mathfrak{p}$. Since $\operatorname{Gal}\left(K_{\ell^{k}} / \mathbb{Q}\right)$ acts on the $\ell^{n}$ th preimages of $\alpha$ in $K_{\ell^{k}}$, and the reduction map is injective, this implies that $\left(\frac{K_{\ell^{k}} / \mathbb{Q}}{\mathfrak{p}}\right) \in \mathcal{C}_{k, n, \ell}$, as desired.

The previous lemma allows us to determine, based on $\left(\frac{K / \mathbb{Q}}{p}\right)$, when preimages exist. The next allows us to determine the group order $\bmod \ell^{k}$.

Lemma 18. Suppose $p$ is a prime number with $p \neq 2,5, \ell$. If

$$
a x+b \in \rho\left(\left(\frac{K_{\ell^{k}} / \mathbb{Q}}{p}\right)\right) \subseteq I\left(\ell^{k}\right),
$$

and $a \not \equiv 1\left(\bmod \ell^{k}\right)$, then $\operatorname{ord}_{\ell}\left(\left|G\left(\mathbb{F}_{p}\right)\right|\right)=\operatorname{ord}_{\ell}(a-1)$. 
Proof. Let $\mathfrak{p}$ be a prime above $p$ in $\mathcal{O}_{K_{\ell^{k}}}$ and let $\sigma=\left(\frac{K_{\ell^{k}} / \mathbb{Q}}{\mathfrak{p}}\right)$. Note that $\sigma\left(\zeta_{\ell^{k}}\right)=\zeta_{\ell^{k}}^{p}$ and define $\epsilon \in\{ \pm 1\}$ by $\sigma(\sqrt{5})=\epsilon \sqrt{5}$. If $\rho(\sigma)=a x+b$, then

$$
\left(\frac{\zeta_{\ell^{k}}^{p}+\zeta_{\ell^{k}}^{-p}}{2}, \frac{\zeta_{\ell^{k}}^{p}-\zeta_{\ell^{k}}^{-p}}{2 \epsilon \sqrt{5}}\right)=\sigma\left(Q_{\ell^{k}}\right)=a Q_{\ell^{k}}=\left(\frac{\zeta_{\ell^{k}}^{a}+\zeta_{\ell^{k}}^{-a}}{2}, \frac{\zeta_{\ell^{k}}^{a}-\zeta_{\ell^{k}}^{-a}}{2 \sqrt{5}}\right) .
$$

Comparing the $x$-coordinates, we obtain that $a \equiv \pm p\left(\bmod \ell^{k}\right)$. Using this fact and comparing the $y$-coordinates give that $a \equiv \epsilon p\left(\bmod \ell^{k}\right)$. Also, $\epsilon=1$ if and only if $\sqrt{5} \in \mathcal{O}_{K_{\ell k}} / \mathfrak{p}$ and so $\epsilon=\left(\frac{p}{5}\right)$. Thus

$$
a-1 \equiv\left\{\begin{array}{lll}
p-1 \quad\left(\bmod \ell^{k}\right) & \text { if } p \equiv 1 \text { or } 4 \quad(\bmod 5) \\
-p-1 \quad\left(\bmod \ell^{k}\right) & \text { if } p \equiv 2 \text { or } 3 \quad(\bmod 5) .
\end{array}\right.
$$

Lemma 8 now yields the desired result.

Now, for $1 \leq t<k$, define

$$
\begin{array}{r}
\mathcal{D}_{k, t, \ell}=\left\{\sigma \in \operatorname{Gal}\left(K_{\ell^{k}} / \mathbb{Q}\right): a_{\sigma} \not \equiv 1 \quad\left(\bmod \ell^{k}\right) \text { and if } n=\operatorname{ord}_{\ell}\left(a_{\sigma}-1\right),\right. \\
\text { then } \left.\sigma \notin \mathcal{C}_{k, n-t+1, \ell}\right\} .
\end{array}
$$

Here $a_{\sigma}$ denotes the coefficient of $x$ in $\rho(\sigma)=a_{\sigma} x+b_{\sigma} \in I\left(\ell^{k}\right)$. Combining Lemma 11. Lemma [17 and Lemma 18, we see that if $p$ is a prime with $p \not \equiv$ $\pm 1\left(\bmod \ell^{k}\right)$, then

$$
\ell^{t} \text { divides }|\alpha| \text { if and only if }\left(\frac{K_{\ell^{k}} / \mathbb{Q}}{p}\right) \subseteq \mathcal{D}_{k, t, \ell} .
$$

Lemma 19. Assume the notation above. For $1 \leq t<k$, we have

$$
\frac{\left|\mathcal{D}_{k, t, \ell}\right|}{\left|\operatorname{Gal}\left(K_{\ell^{k}} / \mathbb{Q}\right)\right|}=\frac{\ell^{2-t}-\ell^{2-k}-\ell^{1-k}+\ell^{1-2 k+t}}{\ell^{2}-1} .
$$

Proof. Noting that $P_{m, r}=P_{m}+r Q_{m}$ we see that $\sigma$ fixes $\ell^{k-n+t+1} P_{\ell^{k}, r}$ if and only if $\left(a_{\sigma}-1\right) r+b_{\sigma} \equiv 0\left(\bmod \ell^{n-t+1}\right)$. It is easy to see that there are no solutions $r$ to this congruence if and only if $\ell^{n-t+1} \nmid b$. Thus, $\left|\mathcal{D}_{k, t, \ell}\right|=\left\{(a, b) \in I\left(\ell^{k}\right): \operatorname{ord}_{\ell}(a-1)=\right.$ $n$ and $\left.\ell^{n-t+1} \nmid b\right\}$. We find that there are $\ell^{2 k-2 n+t-2}(\ell-1)\left(\ell^{n-t+1}-1\right)$ elements of $I\left(\ell^{k}\right)$ satisfying these properties if $n \geq t$, and 0 if $n<t$. Summing from $n=t$ to $k-1$ we get

$$
\left|\mathcal{D}_{k, t, \ell}\right|=\frac{\ell^{2 k-t+1}-\ell^{k+1}+\ell^{t}-\ell^{k}}{\ell+1}
$$

and dividing by $\left|I\left(\ell^{k}\right)\right|=\ell^{2 k-1}(\ell-1)$ gives the desired result.

We are now able to prove Theorem 3 using similar reasoning.

Proof of Theorem 3. Fix a prime $q \neq 2$ and integers $i$ and $j$ with $i \geq j \geq 0$. By Lemma 10, we have $\operatorname{ord}_{q}(Z(p))=\operatorname{ord}_{q}(|\alpha|)$ for $\alpha=(3 / 2,1 / 2) \in G\left(\mathbb{F}_{p}\right)$. Let $k=i+1$ and $p \neq 2,5, q$ be a prime. As in the proof of Lemma 19] we have $\operatorname{ord}_{q}\left(p-\epsilon_{p}\right)=i$ and $\operatorname{ord}_{q}(Z(p))=j$ if and only if when $\sigma \in\left(\frac{K_{q^{k}} / \mathbb{Q}}{p}\right)$,

$$
\operatorname{ord}_{q}\left(a_{\sigma}-1\right)=i \text { and } \operatorname{ord}_{q}\left(b_{\sigma}\right)=i-j
$$

(with the exception that when $j=0$, there is a $q^{n}$ th preimage of $\alpha$ for all $n$ and so $\left.\operatorname{ord}_{q}\left(b_{\sigma}\right) \geq i\right)$. When $i$ and $j$ are both positive, there are $(q-1)^{2} q^{2 k-2 i+j-2}$ pairs of $\left(a_{\sigma}, b_{\sigma}\right)$. When $i=0$, there are $(q-2) q^{2 k-2}$ pairs, and when $i \geq 1$ and $j=0$, 
there are $(q-1) q^{2 k-2 i-1}$ pairs. Applying the Chebotarev density theorem proves the desired result.

Now, we turn to the composite case. Let $m=\prod_{i=1}^{r} \ell_{i}^{s_{i}}$. Take $M=\prod_{i=1}^{r} \ell_{i}^{S_{i}}$ to be a multiple of $m$. The discussion following the proof of Lemma 18 now implies the following statement. If $p \not \equiv \pm 1\left(\bmod \ell_{i}^{S_{i}}\right)$ for all $i, 1 \leq i \leq r$, then $m$ divides the order of $\alpha$ in $G\left(\mathbb{F}_{p}\right)$ if and only if for all $\sigma \in\left(\frac{K_{M} / \mathbb{Q}}{p}\right)$,

$$
\left.\sigma\right|_{K_{\ell_{i}}} \in \mathcal{D}_{S_{i}, s_{i}, \ell_{i}} \text { for all } i, 1 \leq i \leq r .
$$

In the case where $m$ is coprime to 10 , it follows from Lemma 16 that $\operatorname{Gal}\left(K_{M} / \mathbb{Q}\right) \cong$ $\prod_{i=1}^{r} \operatorname{Gal}\left(K_{\ell_{i}^{S_{i}}} / \mathbb{Q}\right)$, and the fraction of elements $\sigma$ that are in $\mathcal{D}_{S_{i}, s_{i}, \ell_{i}}$ for all $i$ is

$$
\prod_{i=1}^{r} \frac{\left|\mathcal{D}_{S_{i}, s_{i}, \ell_{i}}\right|}{\left|\operatorname{Gal}\left(K_{M} / \mathbb{Q}\right)\right|} .
$$

In the case where $m$ is a multiple of $10, \operatorname{Gal}\left(K_{M} / \mathbb{Q}\right)$ is an index 2 subgroup of the direct product and counting is more tricky. For this reason, we now define for $t_{1} \geq 1$ and $t_{2} \geq 1$ :

$$
\begin{gathered}
\mathcal{D}_{k, t_{1}, t_{2}, 10}=\left\{\sigma \in \operatorname{Gal}\left(K_{10^{k}} / \mathbb{Q}\right): a_{\sigma} \not \equiv 1 \quad\left(\bmod 2^{k}\right) \text { and } a_{\sigma} \not \equiv 1 \quad\left(\bmod 5^{k}\right)\right. \\
\text { and if } n_{1}=\operatorname{ord}_{2}\left(a_{\sigma}-1\right), n_{2}=\operatorname{ord}_{5}\left(a_{\sigma}-1\right), \\
\text { then } \left.\sigma \notin \mathcal{C}_{k, n_{1}-t_{1}+1,2} \text { and } \sigma \notin \mathcal{C}_{k, n_{2}-t_{2}+1,5}\right\}
\end{gathered}
$$

For $t_{1}=0$, we omit the condition $\sigma \notin \mathcal{C}_{k, n_{1}-t_{1}+1,2}$.

Lemma 20. Assume the notation above. For $0 \leq t_{1}<k$ and $1 \leq t_{2}<k$, we have

$$
\begin{aligned}
\frac{\left|\mathcal{D}_{k, t_{1}, t_{2}, 10}\right|}{\left|\operatorname{Gal}\left(K_{10^{k}} / \mathbb{Q}\right)\right|} & =\frac{25}{36 \cdot 2^{t_{1} 5^{t_{2}}}}-\frac{5}{6 \cdot 2^{t_{1}} 5^{k}}+\frac{5}{36 \cdot 2^{t_{1}} 5^{2 k-t_{2}}}+\frac{5}{2 \cdot 10^{k}}-\frac{5}{12 \cdot 2^{k} 5^{2 k-t_{2}}} \\
& -\frac{25}{12 \cdot 5^{t_{2}} 2^{k}}+\frac{5}{18 \cdot 2^{2 k-t_{1} 5^{2 k-t_{2}}}}+\frac{25}{18 \cdot 2^{2 k-t_{1} 5^{t^{2}}}}-\frac{5}{3 \cdot 2^{2 k-t_{1}} 5^{k}},
\end{aligned}
$$

when $t_{1}>0$, and

$$
\begin{aligned}
\frac{\left|\mathcal{D}_{k, 0, t_{2}, 10}\right|}{\left|\operatorname{Gal}\left(K_{10^{k}} / \mathbb{Q}\right)\right|}= & \frac{25}{9 \cdot 5^{t_{2}}}-\frac{25}{9 \cdot 5^{t_{2}} \cdot 4^{k}}+\frac{5}{9 \cdot 5^{2 k-t_{2}}} \\
& -\frac{10}{3 \cdot 5^{k}}-\frac{5}{9 \cdot 4^{k} \cdot 5^{2 k-t_{2}}}+\frac{10}{3 \cdot 20^{k}} .
\end{aligned}
$$

Proof. Arguing as in Lemma 19] $\left|\mathcal{D}_{k, t_{1}, t_{2}, 10}\right|$ is the number of $a x+b \in I\left(10^{k}\right)$ that satisfies

$$
\begin{aligned}
& b \text { even } \Longleftrightarrow a \equiv 1,4 \quad(\bmod 5), \\
& a \neq 1 \quad\left(\bmod 2^{k}\right), \quad a \neq 1 \quad\left(\bmod 5^{k}\right), \\
& \operatorname{ord}_{2}(a-1)=n_{1}, \quad \operatorname{ord}_{5}(a-1)=n_{2}, \\
& 2^{n_{1}-t_{1}+1} \nmid b, \text { and } 5^{n_{2}-t_{2}+1} \nmid b,
\end{aligned}
$$

provided $t_{1}>0$. In the case where $b$ is odd, $a \equiv 2,3(\bmod 5)$ and $t_{2}=0$. In the case where $b$ is even and $a \equiv 1,4(\bmod 5)$, there are $2^{k-n_{1}-1} \cdot 4 \cdot 5^{k-n_{2}-1}$ choices 
for $a$ and $\left(2^{k-1}-2^{k-n_{1}+t_{1}-1}\right)\left(5^{k}-5^{k-n_{1}+t_{1}-1}\right)$ choices for $b$. Summing over $n_{1}$ and $n_{2}$ gives

$$
\sum_{n_{1}=t_{1}}^{k-1} \sum_{n_{2}=t_{2}}^{k-1} 4 \cdot 2^{k-n_{1}-1} \cdot 5^{k-n_{2}-1}\left(2^{k-1}-2^{k-n_{1}+t_{1}-1}\right)\left(5^{k}-5^{k-n_{2}+t_{2}-1}\right) .
$$

This sum of four geometric series is easily evaluated to give the stated answer. In the case where $t_{1}=0$, there are again $2^{k-n_{1}-1} \cdot 4 \cdot 5^{k-n_{2}-1}$ choices for $a$ and $2^{k-n_{1}} \cdot\left(5^{k}-5^{k-n_{2}+t_{2}-1}\right)$ choices for $b$. Summing yields the stated result.

We are now ready to prove the main result.

Proof of Theorem 2. Let $m$ be a positive integer with $\operatorname{gcd}(m, 10)=1$ and fix an $\epsilon>0$. Let $m=\prod_{i=1}^{r} \ell_{i}^{s_{i}}$ be the prime factorization of $m$ and note that $\lim _{k \rightarrow \infty} \frac{\left|\mathcal{D}_{k, t, \ell}\right|}{\left|\operatorname{Gal}\left(K_{\ell k} / \mathbb{Q}\right)\right|}=\zeta\left(\ell^{t}\right)$ by Lemma 19. Choose a positive real number $\eta$ small enough so that $1-\frac{\epsilon}{2} \leq(1-\eta)^{r}$ and $(1+\eta)^{r} \leq 1+\frac{\epsilon}{2}$. Now, let $M=\prod_{i=1}^{r} \ell_{i}^{S_{i}}$ be chosen with $S_{i}$ sufficiently large that

$$
\zeta\left(\ell^{s_{i}}\right)-\eta \leq \frac{\left|\mathcal{D}_{S_{i}, s_{i}, \ell_{i}}\right|}{\left|\operatorname{Gal}\left(K_{\ell_{i}^{S_{i}}} / \mathbb{Q}\right)\right|} \leq \zeta\left(\ell^{s_{i}}\right)-\frac{2}{\left(\ell_{i}-1\right) \ell_{i}^{S_{i}}}+\eta
$$

for $1 \leq i \leq r$. Combining the Chebotarev density theorem with the observation that $\ell_{i}^{s_{i}}$ divides the order of $\alpha \in G\left(\mathbb{F}_{p}\right)$ if and only if $\left(\frac{K_{\ell} s_{i} / \mathbb{Q}}{p}\right) \subseteq \mathcal{D}_{S_{i}, s_{i}, \ell_{i}}$ (provided $\left.p \not \equiv \pm 1\left(\bmod \ell_{i}^{S_{i}}\right)\right)$, we obtain that the number of primes $p \leq x$ for which $m$ divides $|\alpha|$ satisfies

$$
\begin{aligned}
-\epsilon / 2+\prod_{i=1}^{r} \frac{\left|\mathcal{D}_{S_{i}, s_{i}, \ell_{i}}\right|}{\operatorname{Gal}\left(K_{\ell_{i}^{S_{i}}} / \mathbb{Q}\right)} & \leq \frac{\#\{p \leq x: m|| \alpha \mid\}}{\pi(x)} \\
& \leq \prod_{i=1}^{r} \frac{\left|\mathcal{D}_{S_{i}, s_{i}, \ell_{i}}\right|}{\operatorname{Gal}\left(K_{\ell_{i}^{S_{i}}} / \mathbb{Q}\right)}+\frac{2}{\left(\ell_{i}-1\right) \ell_{i}^{S_{i}-1}}+\epsilon / 2
\end{aligned}
$$

provided $x$ is sufficiently large. We have that

$$
\zeta(m)(1-\epsilon / 2) \leq \prod_{i=1}^{r}\left(\zeta\left(\ell_{i}^{s_{i}}\right)-\eta\right) \leq d \leq \prod_{i=1}^{r}\left(\zeta\left(\ell_{i}^{s_{i}}\right)+\eta\right) \leq \zeta(m)(1+\epsilon / 2),
$$

which implies that

$$
(\zeta(m)-\epsilon) \pi(x) \leq \#\{p \leq x: m \| \alpha \mid\} \leq(\zeta(m)+\epsilon) \pi(x),
$$

provided $x$ is large enough. Combining this with Lemma 10 proves Theorem 2 in the case where $\operatorname{gcd}(m, 10)=1$. The case where $\operatorname{gcd}(m, 10)>1$ is similar with two notable differences: there is extra complexity in dealing with the primes 2 and 5 using Lemma 20, and Lemma 10 shows that $2 \mid Z(p)$ if and only if $|\alpha|$ is odd.

\section{ACKNOWLEDGEMENTS}

This work represents the master's thesis of the first author, which was completed at Wake Forest University. The authors would also like to thank Pieter Moree for helpful comments. 


\section{REFERENCES}

[1] Christian Ballot, Density of prime divisors of linear recurrences, Mem. Amer. Math. Soc. 115 (1995), no. 551, viii+102, DOI 10.1090/memo/0551. MR.1257079 (95i:11110)

[2] Paul S. Bruckman and Peter G. Anderson, Conjectures on the Z-densities of the Fibonacci sequence, Fibonacci Quart. 36 (1998), no. 3, 263-271. MR.1627443 (99i:11007)

[3] R. D. Carmichael, On the numerical factors of the arithmetic forms $\alpha^{n} \pm \beta^{n}$, Ann. of Math. (2) 15 (1913/14), no. 1-4, 49-70, DOI 10.2307/1967798. MR.1502459

[4] Whitfield Diffie and Martin E. Hellman, New directions in cryptography, IEEE Trans. Information Theory IT-22 (1976), no. 6, 644-654. MR0437208 (55 \#10141)

[5] Helmut Hasse, Über die Dichte der Primzahlen p, für die eine vorgegebene ganzrationale Zahl $a \neq 0$ von durch eine vorgegebene Primzahl $l \neq 2$ teilbarer bzw. unteilbarer Ordnung mod. $p$ ist (German), Math. Ann. 162 (1965/1966), 74-76. MR0186653 (32 \#4111)

[6] Helmut Hasse, Über die Dichte der Primzahlen p, für die eine vorgegebene ganzrationale Zahl $a \neq 0$ von gerader bzw.ungerader Ordnung mod. $p$ ist (German), Math. Ann. 166 (1966), 19-23. MR0205975 (34 \#5800)

[7] Henryk Iwaniec and Emmanuel Kowalski, Analytic number theory, American Mathematical Society Colloquium Publications, vol. 53, American Mathematical Society, Providence, RI, 2004. MR2061214 (2005h:11005)

[8] Rafe Jones and Jeremy Rouse, Galois theory of iterated endomorphisms, Appendix A by Jeffrey D. Achter. Proc. Lond. Math. Soc. (3) 100 (2010), no. 3, 763-794, DOI 10.1112/plms/pdp051. MR2640290(2011d:11120)

[9] J. C. Lagarias, The set of primes dividing the Lucas numbers has density 2/3, Pacific J. Math. 118 (1985), no. 2, 449-461. MR789184 (86i:11007)

[10] J. C. Lagarias, Errata to: "The set of primes dividing the Lucas numbers has density $2 / 3$ " [Pacific J. Math. 118 (1985), no. 2, 449-461; MR0789184 (86i:11007)], Pacific J. Math. 162 (1994), no. 2, 393-396. MR1251907 (94m:11015)

[11] James S. Milne, Fields and Galois theory, v4.22, 2011, Course notes, available at www.jmilne.org/math/.

[12] Richard A. Mollin, Algebraic number theory, CRC Press Series on Discrete Mathematics and its Applications, Chapman \& Hall/CRC, Boca Raton, FL, 1999. MR,1682930 (2000e:11130)

[13] Pieter Moree, On primes $p$ for which d divides $\operatorname{ord}_{p}(g)$, Funct. Approx. Comment. Math. 33 (2005), 85-95. MR2274151(2007j:11131)

Department of Mathematics, Clemson University, Clemson, South Carolina 29634

E-mail address: pcubre@gmail.com

Department of Mathematics, Wake Forest University, Winston-Salem, North CaroLINA 27109

E-mail address: rouseja@wfu.edu 